\section{Black Swans and Bell Curves}

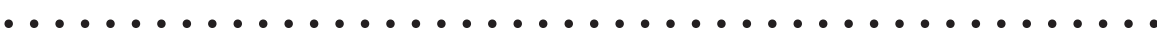

Phenotypic Variation

'Phenotypic Variation (Exploration and Functional Genomics)' Edited by Moyra Smith ISBN-10: 0195379632; ISBN-13: 978-0195379631

Published by: Oxford University Press, 2011

$£ 40.00 \$ 59.95$

...........

Barbara A Jennings

\section{European Journal of Human Genetics (2012) 20, 1202; doi:10.1038/ejhg.2012.118}

$\mathrm{T}$ wo hundred terabytes of data from the Thousand Genomes project is now publicly available through an Amazon Web Services cloud. But the data describing human genetic variation will only be useful when we know how to translate it to improve human health. This partly depends on a comprehensive understanding of the relationship between our genomes and a vast range of phenotypes, be they rare mendelian or common quantitative traits. Moyra Smith helps us in this endeavour; she begins her book on phenotypic variation with cautious words from Lewis Thomas 'Now that we have begun exploring in earnest, doing serious science, we are getting glimpses of how huge the questions are and how far from being answered'. This is an apt quotation for Smith's book, which painstakingly curates an impressive swath of very serious science published in her field of functional genomics up until 2009, and is modest in its claims about how we can currently apply the findings to medicine.

The early chapters provide a detailed context for issues that are subsequently explored with increasing complexity. Genome architecture and structural DNA changes are described that have a functional influence on protein expression and in some cases may mark evolutionarily important stages for humans. For example, comparative genomic studies in humans and primates have identified loci that influence brain function (FOXP2, ASPM) and that have undergone rapid evolution. The influence of alleles under positive natural selection on the human genome is also considered with the concomitant reduction in genetic diversity for specific haplotypes.

Chapter 4, Linkage, Association, and Linkage Disequilibrium, presents data from GWAS (genome-wide association studies) and explores both the rapid progress made and factors that can impede our understanding of associations between genotype and complex phenotypes. Some of the factors are technical; the limitations of data generated from microarrays that were constructed before large-scale mRNA sequencing had revealed the complexity of the transcriptome. Other confounding factors are biological; functional studies reveal a good deal of redundancy because evolutionary processes result in chaotic complex systems, and the co-segregation of some SNPs with disease phenotypes is because of linkage disequilibrium with the causal structural variants within the genome. Further complexity that necessitates the functional plasticity of the human genome is elaborated on in chapters 5-7, which consider transcriptional control; the spliceosome; protein turnover; and the heteroplasmy and mutations that affect the expression of the mitochondrial genome. Aberrant protein folding and impaired autophagy are phenotypic changes associated with ageing and neurodegenerative disease, as are somatic mutations in the mitochondrial genome.

The later chapters consider complex disease phenotypes, including neurobehavioural disorders, malformation syndromes, and late-onset conditions such as Parkinson's disease. Some overarching themes emerge from Smith's detailed review of the results of familial, population genetic, and experimental studies. For psychiatric diseases, the diagnostic categorisation may need to change in the light of genetic findings and an understanding of molecular pathophysiology; a paradigm shift that is already underway in other fields such as oncology. In considering the molecular basis of schizophrenia Matt Ridley's maxim of nature via nurture came to mind because of the putative role of infectious agents in the disease aetiology (schizophrenia-associated markers identified in the MHC region) and the effects of cannabis in its associated cognitive impairment (cannabis sensitivity associated COMT genotypes). By reviewing the common clinical features of Noonan, Costello, and cardio-facial cutaneous syndromes, caused by perturbation of the RAS MAP kinase signalling pathway, we are reminded that we can't understand complex phenotypes without considering how individual proteins function in (networks of) biochemical pathways.

This book provided a deeply satisfying read for the human genetics geek who does not get out of her own narrow research niche nearly often enough. But it is quite dry in places and would be a challenge for the novice. The book has a narrative within and between chapters but the story is far from explicit and may have benefited from more signposting and figures with detailed legends; for example, a diagram of the RAS MAP kinase pathway would have been helpful in chapter 10 .

Perhaps the paucity of emphatic conclusions in this book was deliberate, to illustrate the fact that functional genomics has not yet come of age? The new generation of GWAS, the rapid pace of eQTL (expression quantitative trait loci) mapping and metabolomics, together with the launch of the Human Proteome Project all suggest that a detailed understanding of the relationships between our genomes, intermediate phenotypes, and disease aetiology is finally within sight

BA Jennings is at the Norwich Medical School, University of East Anglia, University Plain, Norwich, UK.

E-mail: B.Jennings@uea.ac.uk 